\title{
Identification of A Neural Mass Model of Burst Suppression
}

\author{
Amirhossein Jafarian $^{1, \dagger}$, Dean R. Freestone ${ }^{3}$, Dragan Nešić ${ }^{2}$, David B. Grayden ${ }^{1,3}$
}

\begin{abstract}
Burst suppression includes alternating patterns of silent and fast spike activities in neuronal activities observable (in micro or macro scale) electro-physiological recordings. Biological models of burst suppression are given as dynamical systems with slow and fast states. The aim of this paper is to give a method to identify parameters of a mesoscopic model of burst suppression that can provide insights into study underlying generators of intracranial electroencephalogram (iEEG) data. An optimisation technique based upon a genetic algorithm (GA) is employed to find feasible model parameters to replicate burst patterns in the iEEG data with paroxysmal transitions. Then, a continuous-discrete unscented Kalman filter (CD-UKF) is used to infer hidden states of the model and to enhance the identification results from the GA. The results show promise in finding the model parameters of a partially observed mesoscopic model of burst suppression.
\end{abstract}

\section{INTRODUCTION}

Burst suppression is a pattern of quasi-silent and fast spike-like activities that is ubiquitous in neuronal recordings, from patch clamp recordings of single neuron dynamics to iEEG data that capture mean collective behaviours of neurons in a cortical column [1],[2]. A biologically-inspired model of a burst suppression comprises slow states, $\theta(t)$, and fast states, $x(t)$, whose interaction is modelled as follows:

$$
\begin{aligned}
\dot{x}(t) & =f\left(x(t), \theta(t), \lambda_{1}\right) \\
\dot{\theta}(t) & =\varepsilon g\left(x(t), \theta(t), \lambda_{2}\right) \\
y_{k} & =H x\left(t_{k}\right)+r_{k}, \quad k=\{1,2, \ldots, n\}
\end{aligned}
$$

where $0<\varepsilon<1$ is constant to ensure the evolution of $\theta(t)$ is slower than $x(t), \lambda_{1,2}$ are constant model parameters, $y_{k}$ relate time-sampled solutions of the system to the neuronal recording (e.g. iEEG data) using operator $H$, and $r_{k}$ as measurement noise, which is given by an additive random IID process. The role of slow states in Equation (1) is regulatory, which can cause fast states to traverse into quasisilent and bursting modes [1]. Mesoscopic models of burst suppression can provide insights into underlying generators of anaesthesia and epileptic seizures [2].

In this paper, identification of a bursting model is carried out using a genetic algorithm (GA) [4] and a continuousdiscrete unscented Kalman filter (CD-UKF) [5]. By using a GA for parameter estimation, the identification problem is formulated as a nonlinear optimisation problem to find a feasible set of parameters to capture a silent-bursting

*This work was funded by the ARC (Linkage Project LP100200571).

${ }^{1}$ Dept. Biomedical Engineering, The University of Melbourne, VIC 3010, Australia

${ }^{2}$ Dept. Electrical \& Electronic Engineering, The University of Melbourne, VIC 3010, Australia

${ }^{3}$ Dept. Medicine, The University of Melbourne, VIC 3010, Australia

†a.jafarian@ucl.ac.uk pattern in the observation data. This amounts to searching for parameters for which the predicted output of the model and observation data are approximately similar (i.e., their accumulative similarity over all data samples is maximised). Using the estimation results from the GA, the CD-UKF algorithm is then employed to enhance results by refining the values of the estimated parameters in the process of inferring hidden states for the data. This amounts to local states estimations of the model and modification of parameters values if necessary.

In Section 2, a brief overview of the neural mass models of burst suppression is given. In Section 3, the two-stage identification method based upon the GA and CD-UKF is explained.

\section{Methods}

\section{A. Neural Mass Model of Burst Suppression}

In this paper, the mesoscopic burst suppression model is the Jansen and Rit neural mass model (JNMM) [6], with some of its internal parameters acting as slow states. The JNMM comprises pyramidal neurons $(p)$, inhibitory $(i)$ interneurons, excitatory $(e)$ stellate neurons, and an external input received by the excitatory neurons [6]. The dynamic of mean activity of each neuronal population is mdoelled by the mean potential-to-firing rate and firing rates-to-potential operators. The mean potential-to-firing rate conversion is given by a sigmoid function $g($.$) that transforms a membrane$ potential $x(t)$ into a firing rate as follows:

$$
g\left(x(t), v_{0}\right)=\frac{2 e_{0}}{\left(1+e^{\zeta\left(v_{0}-x(t)\right)}\right)},
$$

where $v_{0}$ is the firing threshold (when the postsynaptic potential (PSP) achieves $50 \%$ of the maximum firing rate), $\varsigma$ is the slope at the firing threshold, and $2 e_{0}$ is the maximum firing rate of the neural population. The mean firing rate is converted to potential by a linear low-pass filter with impulse response $h(t)=\alpha \zeta t e^{-\zeta t} H(t)$ ( $\alpha$ is the static filter gain proportional to maximum postsynaptic potential, $\zeta$ is synaptic time constant, and $H(t)$ is the Heaviside function). For input firing rates $g\left(x_{i}(t), v_{0}\right)+N(t)(N(t)$ is an external input) into the synapse of population $j$, a post synaptic potential, $x_{j}(t)$ is generated that can be calculated by the convolution integral $x_{j}(t)=\left(g\left(x_{i}(t), v_{0}\right)+N(t)\right) * h(t)$, which can also be written as follows:

$$
\ddot{x}_{j}(t)+2 \zeta_{j} \dot{x}_{j}(t)+\zeta_{j}^{2} x_{j}(t)=\alpha \zeta\left(g\left(x_{b}(t), v_{0}\right)+N(t)\right)
$$




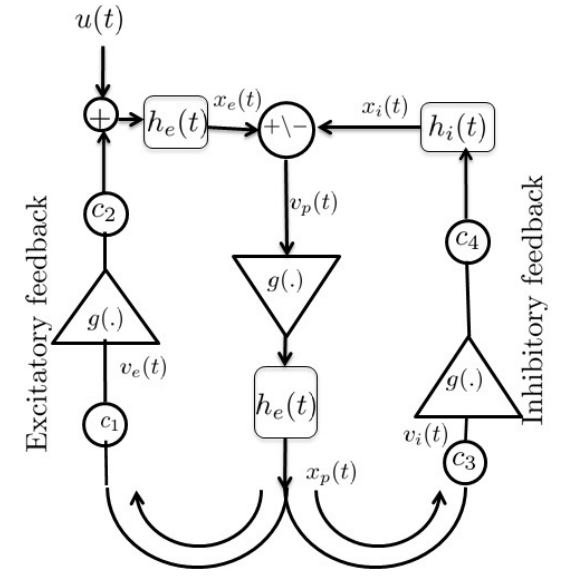

Fig. 1. Neural Mass Model. Simulated iEEG data is the membrane potential of the pyramidal population $v_{p}(t)$. The states are represented by $x_{j}(t)(j=p$ (pyramidal), $e$ (excitatory), $i$ (inhibitory)) as the potentials that are generated at time $t$ by the pyramidal, excitatory and inhibitory populations, respectively. The postsynaptic potentials that are generated in the pyramidal, excitatory and inhibitory populations are denoted by $v_{j}(t)$ ( $j=p, e$, and $i$ ), respectively. The nonlinear function $g($.$) is a sigmoid$ function that converts the mean membrane potentials to the mean firing rate. Each synaptic kernel, $h_{j}(t)(j=e$ and $i)$, is a second-order linear operator that converts the firing rates to a synaptic potential. The model has two excitatory populations, one is pyramidal cells in layer III and IV and the other one models the spiny cells in layer IV. Impulse response of the synaptic transmission of pyramidal and excitatory populations are considered equal to $h_{e}(t)$ in the JNMM. Mean connectivity constants are denoted by $c_{1}, c_{2}, c_{3}$ and $c_{4}$.

which can be written in canonical state-space form:

$$
\left(\begin{array}{c}
\dot{x}_{j}(t) \\
\dot{x}_{j 1}(t)
\end{array}\right)=\left(\begin{array}{c}
x_{j 1}(t) \\
\alpha_{j} \zeta_{j}\left(g\left(x_{b}(t)+N(t)\right)-2 \zeta_{j} x_{j 1}(t)-\zeta_{j}^{2} x_{j}(t)\right.
\end{array}\right)
$$

Activity-dependent homeostasis, where fast dynamics of the membrane potential and the firing rates of neurons are regulated by slow ion currents, is known as a fundamental characteristic of neuronal behaviours. For example, after generation of action potentials, the firing rate drops because of ionic recovery time due to spike-frequency adaptation (SFA) mechanisms. SFA mechanisms can be modelled as changes in the excitability level of a neuron population, which can be represented as negative feedback from firing rate levels to the level of firing threshold as follows:

$$
\dot{v}_{0}(t)=\varepsilon\left(-\gamma v_{0}(t)+\psi g\left(v(t), v_{0}(t)\right)\right)
$$

where $\gamma$ is decay rate, $\psi$ is the parameter that links fast dynamics of the firing rate and slow states, and $\varepsilon$ is ensures that the rate of change parameters is slower than of firing rates (for model identification aim, we merge the $\varepsilon$ with constant parameters $\gamma$ and $\psi$ ). The mathematical model of burst suppression (hereinafter called the slow-fast JNMM) is given by augmenting the slow dynamics of firing thresholds with the mathematical formulations of the JNMM. The full equations of the slow-fast JNMM are:

$$
\begin{gathered}
\left(\begin{array}{c}
\dot{x}_{p} \\
\dot{x}_{p 1} \\
\dot{v}_{p 0} \\
\dot{x}_{e} \\
\dot{x}_{e 1} \\
\dot{v}_{e 0} \\
\dot{x}_{i} \\
\dot{x}_{i 1} \\
\dot{v}_{i 0}
\end{array}\right)=\left(\begin{array}{c}
x_{p 1} \\
\alpha_{e} \zeta_{e} g\left(x_{e}-x_{i}\right)-2 \zeta_{e} x_{p 1}-\zeta_{e}^{2} x_{p} \\
\left.-\gamma_{p} v_{p 0}+\psi_{p} g\left(x_{e}-x_{i}\right), v_{p 0}\right) \\
x_{e 1} \\
\alpha_{e} c_{2} \zeta_{e} g\left(c_{1} x_{p}\right)-2 \zeta_{e} x_{e 1}-\zeta_{e}^{2} x_{e}+\alpha_{e} \zeta_{e} u \\
-\gamma_{e} v_{e 0}+\psi_{e} g\left(x_{p}, v_{e 0}\right) \\
x_{i 1} \\
\alpha_{i} c_{4} \zeta_{i} g\left(c_{3} x_{p}\right)-2 \zeta_{i} x_{i 1}-\zeta_{i}^{2} x_{i} \\
-\gamma_{e} v_{i 0}+\psi_{e} g\left(x_{p}, v_{i 0}\right) \\
y_{k}=x_{e}\left(t_{k}\right)-x_{i}\left(t_{k}\right)+r_{k}, \quad k=\{1,2, \ldots, n\}
\end{array}\right)
\end{gathered}
$$

Equation (II-A) has typical form of slow-fast system in equation (1) with $x_{p, i, e}(t)$ as the fast states, $v_{p, e, i}(t)$ as the slow states, and $y_{k}$ as the iEEG data.

\section{B. Parameter Estimation in Bursting Model}

In this paper, model identification is performed for the parameters of the slow states (i.e., $\gamma_{i, p, e}$ and $\psi_{i, p, e}$ in equation (II-A) and $\lambda$ in equation (1)). These parameters are responsible for generation of paroxysmal transitions.

1) Genetic Algorithm Based Identification of Bursting Model: The GA can be used to optimize high-dimensional and complex cost functions [4]. Identification of a bursting model can be carried out by searching the parameter space $\lambda$ using the GA to minimize the following cost function:

$$
\operatorname{cost}(\lambda)=\left\|\Gamma\left(\left\{y_{n}\right\}_{n=1}^{k}\right), \Gamma\left(\left\{y_{n}^{\lambda}\right\}_{n=1}^{k}\right)\right\|,
$$

where similarity of iEEG time series, $y_{n}$, and the sampled solution of slow-fast NMM, $y_{n}^{\lambda}$, are evaluated using $l_{2}$ norm (shown by $\|$.$\| ) between scaled (to [0,1]$ ) envelope signals (i.e. norm of Hilbert transform), which is denoted by $\Gamma$. The scaled envelope highlights evolution patterns of spikes during bursting mode as well as timing of the burst.

To evaluate the cost function for each candidate set of parameters, the model needs to be simulated. Due to the existence of slow and fast states in the model, the variable step size Runge-Kutta method followed by re-sampling is used to approximate the system dynamics. This is to ensure the numerical error over parameter space does not affect the search directions by GA and accordingly does not affect the estimation results.

\section{Continuous-Discrete Unscented Kalman Filter Based Pa- rameter Tracking}

Due to the nonlinear and multi-scale nature of the bursting model, there are many parameters that may give rise to similar observation data. Therefore, it might be the case that GA finds parameters where the identified model has generated data approximately similar to observation data, but with different underlying generators. To eliminate this effect, the identified model is augmented by correction states, $\gamma_{c}(t)$ and $\psi_{c}(t)$, that are added to the estimated parameters by the GA (i.e, $\hat{\gamma}$ and $\hat{\psi}$ ). The dynamics of correction states is trivial (i.e., $\dot{\gamma}_{c}(t)=0$ and $\dot{\psi}_{c}(t)=0$ ) and they count as model parameters. Therefore representation of the function 


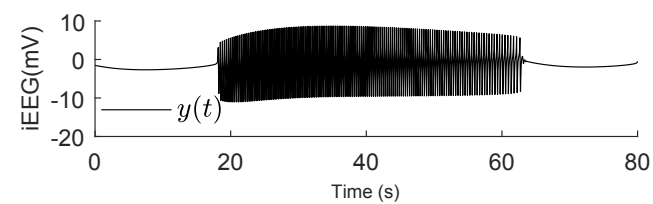

(a)

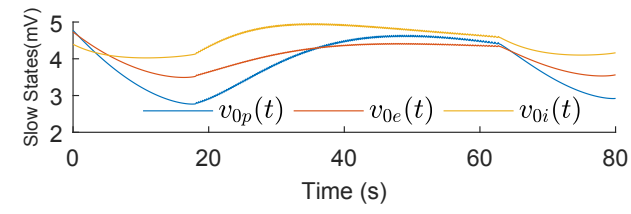

(b)

Fig. 2. Simulated iEEG of slow-fast NMM with transition to and from a seizure. (a) Simulated EEG data, $y(t)$, generated using slow-fast NMM. (b) Time series of firing thresholds of the pyramidal, $v_{0 p}(t)$, excitatory, $v_{0 e}(t)$, and inhibitory, $v_{0 i}(t)$, populations.

$g($.$) remains unchanged. The equation of the new model is:$

$$
\begin{aligned}
& \dot{x}(t)=f(x(t), \theta(t)) \\
& \dot{\theta}(t)=\varepsilon g\left(x(t), \theta(t), \hat{\gamma}+\gamma_{c}(t), \hat{\psi}+\psi_{c}(t)\right) \\
& \dot{\gamma}_{c}(t)=0 \quad \& \quad \dot{\psi}_{c}(t)=0 \\
& y_{k}=H x\left(t_{k}\right)+r_{k}, \quad k=\{1,2, \ldots, n\}
\end{aligned}
$$

The CD-UKF, which is adapted for filtration of continuousdiscrete dynamical systems, is employed to infer the hidden states in the model (8), given the observation data. Using CD-UKF ensures state estimation accuracy of slow and fast states in the model for a fixed sampling rate of observation data. The CD-UKF compensates for parameter errors, differences between estimated model and dynamics of the data in equation (8) by changing the values of the correction states. CD-UKF tracks the model states and tries to keep the evolution of the correction states at constant levels. However, in cases where there is a mismatch between dynamics of the model and data, the CD-UKF changes the values of the correction states to capture the observation iEEG data.

\section{RESULTS}

\section{A. Observation Data}

To test the proposed approach, simulated iEEG data using the slow-fast NMM in equation (1) is generated. The model is simulated using the variable step size Runge-Kutta method for 80 seconds. Then, the solution is re-sampled to generate an observation signal for the identification task with $80 \times 10^{3}$ samples (this is equivalent to a sampling rate of $1000 \mathrm{~Hz}$ ). The simulated iEEG output of the model is given in Figure 2. The simulated data is also contaminated with low level observation noise (as expected in iEEG recroding). All firing thresholds are reduced before initiation of bursting modes and increased during bursting activities toward the end.

\section{B. Estimation Results Using GA}

The parameters of the slow states were estimated using the simulated data. Envelope distance is employed as the cost function, the mutation rate is equal to 0.3 , crossover

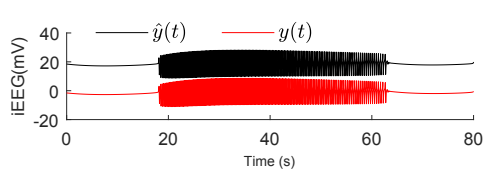

(a)

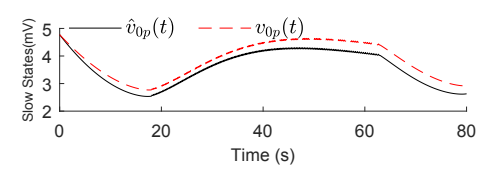

(b)

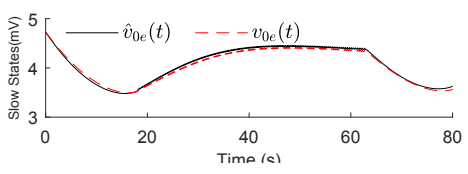

(c)

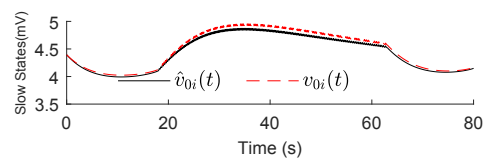

(d)

Fig. 3. Comparison between slow-fast NMM and estimated model using the GA. (a) Observation iEEG, $y(t)$, and estimated EEG outputs of the identified slow-fast NMM, $\hat{y}(t)$, with parameters. An offset of $12 \mathrm{mV}$ added to the estimated iEEG for better visualisation. Estimated and actual slow dynamics of firing thresholds of the (b) pyramidal population, (c) the excitatory population, and (d) inhibitory population.

0.7 , and the initial population is 200 . The mutation value of 0.3 allows the GA to search in a wide range of search space for several generations and not be trapped in local minima. The criterion for which the GA terminates the estimation was when the average change in fitness values for 20 generations remained less than a tolerance of $10^{-6}$. The results in Figure 3(a) show that there is a slight difference between the time of initiation of seizures in the estimated iEEG and observation data. The dynamics of the estimated firing thresholds of excitatory and inhibitory populations are relatively similar to the actual slow dynamics of the generative model, as shown in Figure 3(c) and (d). However, there is an offset difference between the estimated and actual slow dynamics of firing thresholds in the pyramidal population, as shown in Figure 3(b). Observation of estimated slow states shows that, in order to improve the estimation results, compensating for these mismatches is essential for some applications, such as finding optimal performance in the treatment of epileptic seizures or monitoring the condition of the brain during anaesthesia [3].

\section{Estimation Enhancement Using CD-UKF}

The CD-UKF is employed to infer hidden states of the identified model which is augmented with correction states for the observation data. As shown in Figure 3, before the time $20 \mathrm{sec}$, both observation data and the slow-fast model generate silent activity. Therefore, the CD-UKF can estimate the states without too much error. During this period, the dynamics of parameters are almost stationary, as shown in Figure 4. However, at the time $20 \mathrm{sec}$, a large error 


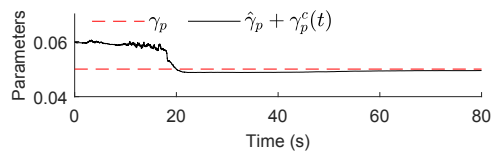

(a)

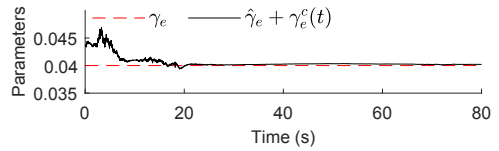

(b)

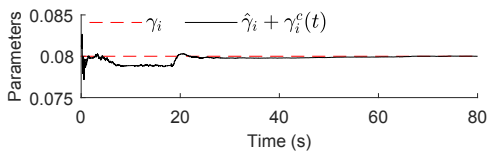

(c)

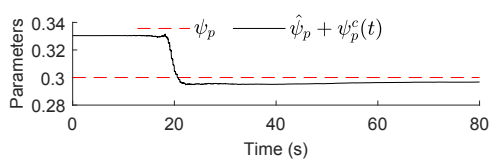

(d)

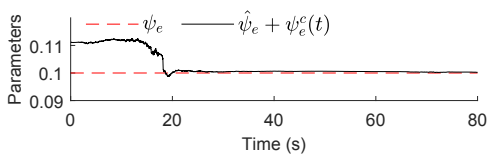

(e)

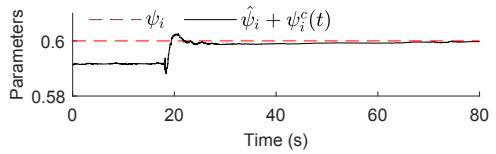

(f)

Fig. 4. Parameter estimation enhancement using UKF. The enhanced estimation of (a) $\gamma_{p}$, (b) $\gamma_{e}$, (c) $\gamma_{i}$,(d) $\psi_{p}$, (e) $\psi_{e}$, (f) $\psi_{i}$. Initial values for parameters are taken from GA-based parameter estimation.

appears in all parameter values. The reason is that the timing of seizures is different between the model and the data. Therefore, the CD-UKF tries to compensate for this inadequacy by changing the correction states. Therefore, we see a relatively sharp jump in the value of the parameters at time $20 \mathrm{sec}$. After the tracking error at the jump point, CD-UKF synchronises data well, and the model and the value of parameters remain unchanged. At the second jump points in the data where data switch from bursting mode into silent activity mode, CD-UKF does not change the values of correction states as the values of parameters converge to their actual values. The mean values of parameters in interval $[25,80]$ can be considered as the estimation outcome, which is very close to the actual values of the parameters. The enhanced estimation of parameter estimation is shown in Figure 4, and the results from the tracking of the slow states are shown in Figure 5.

\section{CONCLUSIONS}

The hybrid estimation based on using GA and CD-UKF is presented to capture underlying mechanisms of burst suppression in the class of slow-fast NMM. By using the

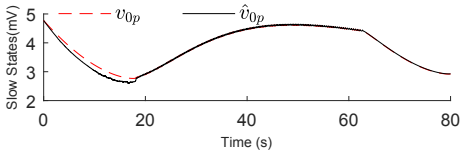

(a)

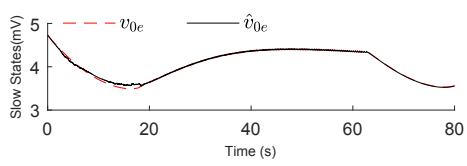

(b)

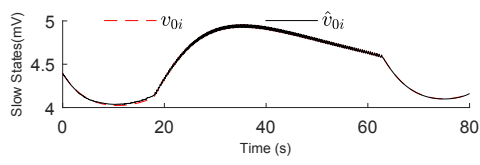

(c)

Fig. 5. The estimated Slow states. (a) Estimated, $\hat{v}_{0 p}$, and actual, $v_{0 p}$ slow evolution of firing thresholds in pyramidal cells. (b) Estimated, $\hat{v}_{0 e}$ and actual, $v_{0 e}$, slow evolution of firing thresholds in excitatory populations. (c) Estimated, $\hat{v}_{0 i}$, and actual, $v_{0 i}$, slow evolution of firing thresholds in inhibitory populations.

GA, parameter estimation is reformulated as an optimisation problem. Results show that the identified model using the GA can generate data similar to observed data; however, it is not guaranteed that estimated parameters converge to actual parameters. This is because there are several feasible parameters that give rise to similar data and in effect the GA might converge to parameter sets that are not necessarily the same as actual parameters. We showed that by using the CD-UKF, the estimation results from the GA can be enhanced. This is due to the fact that CD-UKF can infer and modify estimation of model states sample by sample and, therefore, by using new samples from observation data, the CD-UKF can learn to synchronise data and the model by changing the values of parameters. The proposed approach can be applied to identify parameters of bursting models for instance to investigate underlying distribution of inhibition in post seizures burst suppression. The identified model can potentially be tested to find optimal parameters of electrical stimulation that suppress seizures.

\section{REFERENCES}

[1] S. Coombes and P.C. Bressloff, Bursting: the genesis of rhythm in the nervous system, World Scientific, 2005

[2] D. R. Freestone, D.Nešić, A. Jafarian, and D. B. Grayden, A neural mass model of spontaneous burst suppression and epileptic seizures, Annual International Conference of the IEEE Engineering in Medicine and Biology Society, 2013.

[3] D. Liley and Walsh, The mesoscopic modeling of burst suppression during anesthesia, Frontiers in computational neuroscience, Vol 7, 2013.

[4] D. Simon, Evolutionary optimization algorithms. John Wiley \& Sons; 2013 Jun 13.

[5] S. Sarkka, On unscented Kalman filtering for state estimation of continuous-time nonlinear systems, IEEE Transactions on automatic control, Vol 52, Num 9, pp.1631-1641, 2007.

[6] B. Jansen, and V. Rit, Electroencephalogram and visual evoked potential generation in a mathematical model of coupled cortical columns, Biological Cybernetics, vol. 73, pp. 357-366, 1995. 